\title{
A POST-GRADUATE LECTURE ON "FREQUENCY OF MICTURITION."
}

\author{
BY H. P. WINSBURY-WHITE, F.R.c.s.
}

Surgeon, St. Paul's Hospital.

LADies and GentLemen: If there is one disturbance of micturition which at the same time is more common than the others, gives rise to much general perplexity as to its cause, and which, in the majority of the cases responds well to treatment, it is increased frequency of micturition.

For the purposes of this lecture I intend to treat the subject in an essentially practical way, and the conclusions which I shall give you will be those which are based on my personal experience. I shall deal with the subject under the headings of men, women and children.

First then, as to men. I am bound to mention acute urethritis when dealing with frequency. Where there is an acute and copious purulent discharge, the diagnosis is simple and obvious. But I want to mention especially that there are a number of cases of acute urethritis in which the discharge is very slight, or such may be absent. This impresses us with the necessity of making a very careful examination, and commencing this in the right manner. When we encounter a case complaining of frequency of micturition, we must be careful to inspect the external genitalia before the patient passes water. If he is allowed to pass urine first, we will have lost the opportunity of making our diagnosis if there is a mild urethritis. Often in mild cases we see a little discharge, and sometimes the patient is aware of this, at other times he is not. If a discharge is not found, we must ask the patient to pass his water into two glasses. If we find pus or debris in the first glass and none in the second, the patient has urethritis, thus the diagnosis is made. If we have not proceeded in this way, we might order a cystoscopy, which could have been avoided by a proper examination.

Now for chronic urethritis and infection of the genitals. These conditions may exist without there being any reason to believe that there has been gonorrhœe. These changes are the commonest causes of frequency of micturition of mild degree. They are often combined with other symptoms, such as aching across the lower part of the back, or over the bladder region, or in the perineum, or in the groins. And usually there is no urethral discharge. In some there is a mild gleet. Such a patient is often subject not only to frequency of micturition of a mild and chronic type, but also to attacks of more acute trequency. We must start very carefully in our examination of such a patient. Let us begin by palpating the testes and epididymes; it is common to find a little thickening of one or both where there is a definite complaint in mild frequency. When urethroscoping such patients we may see a stricture, i.e., an advanced stage of chronic urethritis. But the stage of chronic urethritis which precedes this is such that there are only a few scars about the follicles, or there may be a lunule crossing the urethra in a transverse direction. The two-glass test is not so reliable in chronic urethritis, but it is fairly so, and if there is doubt about such a case, a second examination of the urine in two glasses after the passage of a sound, or after prostatic massage, will generally give the necessary information, i.e., there will be débris in the first glass if there is urethral or prostatic involvement. We

1 Delivered at St. Paul's Hospital, on Wednesday, May 3, 1933. 
may safely say that in every case of chronic urethritis evidence can be obtained that the prostate has been implicated as well.

How do we treat a case which falls into the above category? The best way to proceed I believe is to pass a large sound occasionally, so stretching up the follicles in the urethra and prostate. This treatment causes these structures to drain in such a way as to afford some, if not complete, relief from the symptoms.

Next we come to urethral stricture, which is simply an advanced stage of chronic urethritis; it is due to a band of scar tissue which owes its situation to the fact that there has been a long continued inflammation in a follicle near by. The inflammation from this will spread all round the urethra if the process continues for a sufficient length of time. To diagnose a stricture one passes an instrument and notes the obstruction in the urethra. But there is a mild type of stricture in which the obstructing scar tissue is so delicate that a bougie might easily split it without giving an indication of its presence. In order to locate such strictures definitely one should get into the habit of using an anterior urethroscope. If an air distension instrument is employed, it must never be used immediately after any other urethral instrument. It is dangerous to do so. Many strictures are so slight that a small-calibre bougie will not detect them.

I shall now say something about fibrous obstruction at the neck of the bladder, a very common cause of frequency of micturition; it owes its origin, as do the other conditions I have mentioned, to inflammation in the past. I am talking of the results of inflammation of the genitals which are not necessarily gonococcal. It is a postinflammatory condition involving the glandular tissue deep to the vesical trigone in the vicinity of the internal urinary meatus. Inflammation there will ultimately cause fibrosis, sometimes with constriction or a raising of the outlet of the bladder above the ievel of the vesical trigone. Research work has been done on post-mortem material as to this condition, and it shows that it is the whole locality which is involved in the inflammatory change, the prostate, seminal vesicles, verumontanum, the internal meatus and often the vesical trigone too.

How do we diagnose the condition? The patient ustually complains that micturition is frequent and difficult. Other conditions such as a simple enlargement of the prostate and a urethral stricture must of course be excluded. We do a rectal examination and find there is no simple enlargement of the prostate, and we examine the urethra in one of the ways I have described, and we find there is no urethral stricture. Then we examine the patient to eliminate if possible, disease of the central nervous system. But there is a further test which we have to do; after the patient has emptied his bladder as well as he can, we pass a catheter to ascertain whether there is any residual urine. If this is present we are left with the almost certain probability that the patient has an obstruction at the vesical neck. This condition is common, as are all the conditions I have mentioned. We can cystoscope the patient and there is usually found some trabeculation and a retrotrigonal pouch. We may see that the intramural portions of the ureters are prominent as well ; this change represents hypertrophy. It is very interesting in these cases to take a skiagram. In all the cases of chronic genital trouble there is a tendency ultimately, perhaps after a few years, to the formation in the pelvis of phleboliths. They are little rounded bodies with very clearly defined margins, and they lie in the lines of the ureters, and sometimes they cause trouble in discriminating them from ureteric stones. There are calcified glands in the hollow of the sacrum 
and on the posterior abdominal wall which can sometimes be seen as high as the renal hila. These changes are the result of inflammation, and indicate the path of lymphatic spread up the posterior abdominal wall. With regard to X-rays, if one does intravenous pyelography in some of these cases of chronic infection about the neck of the bladder, there is often a little dilatation of the upper part of the ureter and renal pelvis without a corresponding dilatation of the lower part of the ureter. This is not explained by back pressure which would result in the whole ureter being involved. The explanation is that there is a lymphatic spread outside the ureter, and a slow involvement of the kidney and the upper end from the lymphatic tissue at the level of the renal hilum.

With regard to the treatment of these cases of fibrous obstruction at the neck of the bladder, where the quantity of residual urine is small-perhaps $2 \mathrm{oz}$. or less-it is very satisfactory simply to pass a large sound from time to time, to stretch the internal meatus, and in this way such a patient can be adequately treated.

There is sometimes a definite obstruction in the prostatic urethra itself, at others at the internal urinary meatus, and we appreciate this by noting where the point of the instrument is, as the obstruction is met by it. There is a type in which there is no obstruction at any one point, but one notices that there is an elastic resistance to the effort to depress the shaft after the point has entered the bladder, and this means that there is a fibrous change throughout the prostate. This variety is not so easy to deal with by dilatation. Although I say that the conditions I have just described are postinflammatory, there is also present a mild degree of existing inflammation; there is indeed a latent mixed infection in this locality, and when the condition remains untreated the patient is apt to suffer from attacks of cystitis; the latter tend to become worse as time goes on, and the urine ultimately becomes chronically loaded with pus. This is one of the conditions which lead to chronic Bacillus coli infection of the urine.

The worst cases are those in which the urine is badly infected and there is much obstruction ; they do not respond to intermittent dilatation. In my opinion, the proper treatment in these circumstances is transurethral resection of the obstruction at the bladder neck. Pieces of obstructing tissue may be satisfactorily removed by open operation through the suprapubic route. Some have tried prostatectomy in these cases, but it is very unsatisfactory, as there is no proper line of demarcation along which to carry out the enucleation and there is much periprostatic fibrosis with the result that there is a good deal of hæmorrhage and shock. But transurethral resection is an excellent and easy remedy. This procedure, however, is associated with the same risks as prostatectomy. The apparatus used is a cutting instrument, which is actuated by a high-frequency current through a special electrode, and one can remove large pieces of tissue each time one cuts so that there is left a considerable raw area at the neck of the bladder. Therefore, when the general condition of the patient has been lowered by his urinary trouble and there is a good deal of pus and residual urine, a cystostomy should be carried out as a preliminary. Patients have died of pulmonary embolus, pyelonephritis, and pneumonia, following the operation, as a result of not recognizing the necessity for taking this precaution. When done in one stage I would not discharge the patient from hospital after two or three days, as I hear is done in some places. The urine is loaded with pus and débris, and often with blood, for ten or fourteen days afterwards. These patients should be kept in bed and an indwelling catheter provided where necessary while the convalescence is proceeding. 
Simple enlargement of the prostate. The diagnosis is easy in 95 per cent. of the cases by rectal examination, where there is a uniformly enlarged elastic prostate, and the patient has nocturnal frequency. It is not as a rule necessary to cystoscope these patients. To cystoscope in a general way these cases of simple enlargement of the prostate is to get, every now and then, some trouble which would never have occurred otherwise; one may precipitate a case into one of acute retention, or pyelonephritis may be induced. The only cases of simple prostatic enlargement in which I pass the cystoscope are those in which hæmaturia is predominant, for I have had six or seven cases of simple enlargement of prostate which have been complicated by bladder growth, and it is unpleasant to make that discovery on the operating table. And there is the occasional case, in which one is not sure whether it is an adenomatous enlargement or not, and there is the possibility of the middle lobe projecting into the bladder. In one's consulting-room, or in the out-patient department, there is no harm in passing a small calibre catheter to test the residual urine provided the bladder is not distended. I have had no harm arise from proceeding in this way. The difference between passing a soft small-sized catheter and a cystoscope is that with the latter much tension and pressure may be required to manipulate the instrument into position. I would use a No. I6 Coudé French gum-elastic instrument.

Then we have malignant disease of the prostate, which, I am sorry to say, is usually easy enough to diagnose in most cases by rectal examination. In this there is a characteristic hardness, not only in the prostatic region, but also involving the bladder and the seminal vesicles. Occasionally one meets with an instance of an early case where the diagnosis is not certain on making a rectal examination. When such uncertainty arises we should have an X-ray picture taken, because, even in some of the early malignant prostate cases there are characteristic changes in the bony pelvis. If there has been doubt about the diagnosis and there are these bony changes present we need no longer hesitate in deciding that the case is malignant. Occasionally one sees a patient with so widespread a prostatic change from inflammation that it not only involves the prostate gland, but has also spread into the pelvic floor and gives rise to a pelvic cellulitis of a chronic type. Occasionally this condition is somewhat difficult to discriminate from a malignant change. In such a case, if carcinoma is present one will almost certainly find by $\mathrm{X}$-ray examination some change in the bones.

Now we will consider frequency in women. Acute urethritis and acute cervicitis in which the discharge is copious and purulent, is simple enough to diagnose ; but, here again, in women more than in men, we get many cases of acute urethritis in which no discharge has been noticed by the patient because it is so scanty. If we do not proceed carefully in our personal investigation of such a case, putting the woman into the lithotomy position and taking smears where necessary, we shall be liable to miss the real cause of the symptoms. And do not think that because the gonococcus cannot be found, the patient has not got acute urethritis or acute cervicitis : many are not gonococcal in origin. One should consider whether other signs of acute inflammation such as redness, swelling, \&c., are present.

Chronic urethritis and cervicitis in relation to frequency of micturition in women give rise to a large number of cases. My experience is that this group covers most of the cases of frequency in the female, and that the true source of the symptoms is constantly being 'overlooked. Moreover, these conditions are an important predisposing 
cause of $B$. coli infection of the urinary tract. Last year I collected the records of 215 cases of frequency of micturition in women whom I had had under my care and whose cases I had thoroughly investigated, and I found that in those in which the presence of organisms was identified in the urine, 53 per cent. had chronic cervical disease of some kind. You must not think these are all post-gonococcal: some of them are, but it is impossible to know which are and which are not. Some of them have followed in the course of a simple infection and some are post-puerperal in origin. Frequency of micturition may be due to a cervical condition independently of any urethritis, infection having spread to the bladder by way of the lymphatics: there are lymph channels connecting the cervix uteri to the floor of the bladder. I have verified this fact by some experiments I carried out with guinea-pigs. It is of no use to try to clear up a case of cystitis which is associated with cervicitis by treating the bladder and neglecting the cervix.

What are the clinical features of these cases of chronic urethritis and chronic cervicitis which are so common that they provide the bulk of the cases of frequent micturition in women? The earliest symptoms are attacks of frequency at long intervals from each other. An attack may last a week or ten days and then it may not happen again for three or four years. Such a history may persist over a number of years, and ultimately the woman develops chronic frequency. Renal symptoms as a complication may develop even at an early stage. I want you to remember that many a case of acute pyelonephritis which we are called to see is simply one of ascent of infection from below, a fact which is frequently unsuspected. My experience has been that in the great majority of cases of acute pyelonephritis the renal inflammation is the result of an ascending infection from one of the situations I have mentioned. But the kidney condition at such a time occupies the clinical picture, so that one does not think of the other condition. After the acute attack has passed an examination of the lower parts of the genito-urinary apparatus should be carried out.

In speaking of chronic cervicitis, there are several varieties which we must recognize on inspection. First there is the type with simply some muco-purulent discharge, which may be so scanty as not to have attracted the patient's notice, but is obvious on inspecting the cervix with a speculum. Secondly, there is the type in which the discharge is clear, but the vaginal aspect of the cervix is eroded. Sometimes the erosion is seen to be extensive and it may be accompanied also by a muco-purulent discharge. As to the organism which one finds in the cervix in these cases, it is generally $B$. coli, or staphylococcus, or there may be diphtheroid bacilli, i.e., the bacteria are not specific.

With regard to the urethra, in the 2I5 cases of frequency in women whom I have investigated, there was urethral inflammation present in 64 per cent. in which organisms were present in the urine. So that in these 215 women with frequency and organisms in the urine 53 per cent. had chronic cervical disease, and 64 per cent. had chronic urethral disease. Thus these two conditions are not uncommon in cases of frequency of micturition.

How do we determine that the urethra is at fault? The external urinary meatus may appear to be normal, and yet the patient has chronic urethritis. In some cases, by pulling the labia apart and looking carefully, one finds small granulations projecting from the external urinary meatus, and at other times a caruncle is present; both are manifestations of a chronic inflammatory process. In others the orifice of the meatus 
is reddened because the glands near the opening are chronically inflamed, and often the orifice, instead of being reddened, is simply retracted. In the ordinary way, the external urinary meatus is above the general surface of the vestibule, but in many of these cases it is below the surface, a sign of a chronic inflammation. In others when one attempts to pass a cystoscope one cannot get the point of the instrument into the urethral orifice. This indicates the presence of a meatal stricture. There is another type of case in which none of the things I have been describing are obvious on inspection, but one notices on passing into the urethra an instrument which should easily enter the bladder, that it is gripped. This means that there is a stricture or a generalized fibrous narrowing of the whole tube. If one urethroscopes the patient one may see patches of inflammation about the channel. It is an interesting point about many of these cases that the urine is perfectly clear on naked-eye inspection, although marked frequency is present. I would go so far as to say that it is not safe to accept a urine report which states that few or no pathological elements of an inflammatory nature are present, as an indication that infection is not the cause of the patient's symptoms. There are numbers of cases with chronic inflammatory.conditions in the urethra in women in which the urine is clear on inspection, and only a pus cell or two is found even by a skilled bacteriological examination.

These cases lead ultimately to $B$. coli infection. I have catheterized the ureters in a large number of these women. In my early days it was my belief that the coliform infection came from the kidney, but I am now certain that it does not, but that it comes from the base of the bladder. In about $5^{\circ}$ per cent. of a large number of cases I examined there was no evidence of infection in the ureteric catheter specimens, neither pus nor organisms, yet on the same occasions pus and organisms were obtained from the bladder specimens. These findings are corroborated by all those who have examined large numbers of cases in this way. This means that the infection starts below. In examining these chronic cervical and urethral cases we should always do a bi-manual examination. It is usual to find some little change in one or other of the lateral fornices. Often the fornix on one side is a little thickened and not so elastic as the other side; and when one presses on the cervix in one direction it is tender, or it is hard in consistence or it does not move as freely in one direction as another. These are characteristics of chronic cervicitis. The thickening in the lateral fornix is due to a mild pelvic cellulitis, the infection having spread from the cervix. When one cystoscopes these patients it is common, where there is urethritis, to find that the urethra is tender. The presence of this sign may be regarded as of special significance, as the normal female can stand the passage of the cystoscope without complaining of pain.

We frequently find that the whole bladder is normal in appearance, the blood-vessels standing out clearly, and there is the pale appearance of the mucosa, until we look at the trigone. If we do not specially examine this part, it is easy to miss any inflammation which may be present. A wide range of inflammatory changes varying from hyperæmia to ulceration is seen in the course of examining many cases. There may also be a diffuse cystitis which is simply a spread from the trigone, and when the former occurs its source from the latter situation is often obscured. When the urethra is chronically inflamed in such cases, the cystoscope usually shows inflammatory tags on the margin of the internal urinary meatus, generally on the floor.

I have mentioned the use of X-rays in regard to the male. This examination is 
equally helpful in the female. Many of these patients have renal symptoms, and a skiagram has been ordered because urinary stone has to be considered. The kidney becomes involved in the infection and there may be definite renal colic without the presence of stone. Phleboliths and calcified glands are noted frequently on the skiagrams as in the male.

Here is another interesting point bearing on the relationship between upper and lower urinary tract trouble in these cases. I can recall at least six women in the last two years with the types of cervicitis I have described who have passed stones down the ureters, and whose stones, I think, owed their origin to the chronic inflammation in the pelvic floor, which in its turn influenced the kidneys to form calculi as a result of an upward spread of infection. I am more and more inclined to the belief that even mild chronic inflammatory pelvic conditions are important ætiological factors in many cases of renal calculus, in both men and women. I certainly have been impressed to find the two groups of conditions frequently associated in a number of cases. Shall we regard these findings as merely coincidences or has the association any significance? I think it has.

With regard to renal changes as shown by pyelography, in the male, as in the female, there is a tendency to a mild dilatation of the renal pelvis and upper part of the ureter. Clubbing of the calyces is another early sign of chronic renal disease as a result of a chronic mild infection of the lower urinary tract and genitalia. In many cases, one can mark out the outline of the ureter by intravenous urography and it seems normal, except that in the upper two inches or so there is a mild dilatation. I think these changes are the result of a perivascular invasion of the kidney and the upper part of the ureter by way of the lymphatics, the latter entering round the blood-vessels as they pass into the renal hilum.

As to treatment, in the acute cases one carries out irrigations of the bladder and the urethra with potassium permanganate. I am against putting instruments and lotions into the cervix in the presence of acute inflammation, and I have found good results from using a mild antiseptic with glycerine as a base : such a mixture as glycerine and flavin, glycerine and ichthyol, or even glycerine alone, is packed into the vagina. One gets the hygroscopic action of the glycerine which promotes drainage from the inflamed part.

With regard to the treatment of chronic cases in women, for years I treated the cervix with various antiseptics on swabs, \&c., but I have now given that method up, especially where there is erosion. It is most satisfactory to dilate the cervix, under an anæsthetic, up to a No. I 2 Hagar and, with the actual cautery, to make four quick linear sears on each wall of the endocervix and four radiating light cauterizations on the vaginal aspect of the cervix. It is remarkable the way in which a chronic erosion of the cervix which has for years resisted every attempt at treatment will show in a short time ingrowing healthy epithelium all round. In many cases the treatments need only be carried out once. After doing the cauterization of the cervix, one dilates the urethra quite considerably. I have never had any incontinence nor any trouble from treating the urethra in this way, whereas the improvement in the frequency is as a rule very striking indeed; where it is only the urethra that requires attention, the best treatment is intermittent dilatation with sounds increasing gradually in size until the largest is attained, and then to extend the intervals between the treatments from two weeks to three, and even six months. 
In the $2 I_{5}$ cases of frequency of micturition in women I have spoken of, 5 per cent. had urethral stricture. In a woman a urethral stricture is often an advanced one when first discovered. This is because the condition, not being suspected, goes untreated for a long time. I have had several cases in which a filiform bougie could not be passed without an anæsthetic. The treatment of urethral stricture in a woman is regular intermittent dilatation.

Some women have frequency from some degree of vesico-vaginal displacement and prolapse of the pelvic organs, and they commonly develop B.coli infection of the urinary tract. One can rectify the displacements, but the frequency and urinary infection generally persist. There is something more subtle in the pathology than the mere mechanical factor, because repair of the displacements often has little or no beneficial effect on the urinary tract symptoms. These are the worst cases of frequency in women to treat. Often these patients have a chronic condition of the cervix and of the urethra as well. A pelvic tumour will also predispose to chronic infection, probably by way of the pelvic lymphatics to the bladder. In ny 215 cases of frequency in women 18 per cent. had pelvic disorders other than cervical disease associated with their frequency.

The substance of the remarks I have made up to the present is, that in both sexes the great majority of cases of frequency depend upon infection which has settled in the lower urinary tract. The whole group of these cases respond very well indeed to treatment if one gets to the real source of the trouble and commences the treatment properly. If one waits until the patient has got chronic cystitis, with fibrosis of the bladder walls, so that the vesical cavity will only hold a few ounces of urine, the patient has passed the stage when much help can be given. Some relief, however, can be obtained by vesical lavage.

In vesical growth the outstanding feature is hæmaturia. Many women with frequency suffer from acute attacks of cystitis, with hæmaturia, and one must inquire carefully as to whether the blood comes at the end of the act of passing water. Terminal hæmaturia is much more commonly a symptom of an intense inflammation of the trigone than of anything else.

Vesical calculus causes marked frequency sometimes leading to slight incontinence in certain cases. One can settle the diagnosis concerning this question with the cystoscope or by X-rays. In men one may find a vesical diverticulum which causes frequency owing to a gradual infection which supervenes as a result of the residual urine which collects.

Urinary tuberculosis must be remembered with regard to chronic frequency. The condition is generally an example of the spread of infection from the upper urinary tract to the bladder. We must investigate the case by cystoscopy and the passing of ureteric catheters, inoculation of guinea-pigs, and so on. Intravenous pyelography has been very helpful in these cases. Usually a tuberculous case is easy to diagnose if the condition is thought of and the proper means of investigating are used.

It is very important to be able to investigate cases of frequency in children. In them, though the causes of frequency are slight, they are definite and if not carefully sought they can easily be overlooked. A boy with frequency and phimosis has possibly some balanitis, and he should be circumcised. A boy at any age may scream every time he passes his water, which he does frequently, and occasionally a little blood appears at the end of the act of micturition. Most of us were brought up in the belief that terminal hæmaturia is an indication of stone, but it is more frequently a result of 
inflammation; in a large series there would be fifty cases of simple inflammation of the trigone to one of stone as a cause of terminal hæmaturia. In many children who have been circumcised and who are affected in this way we will often find there is a small crust over the external urinary meatus; this is the dried discharge of chronic urethritis, and it is generally associated with an unusually small external meatus. Another interesting fact about this type of case is that often the epididymes are thickened showing that the infection has spread to the posterior urethra and thus to the epididymis. Sometimes the process goes even further and the involvement of the epididymis gives rise to hydrocele. Hydrocele in young children should always be considered in the light of this pathological process. I saw a case recently which not only had both epididymes thickened, but there was bilateral hydrocele as well. B. coli were found in the urine and there was atresia of the external urinary meatus which was covered with a dry crust. The child was suffering from frequency of micturition, dysuria and terminal hæmaturia. Chronic enuresis cases who also suffer from frequency are scmetimes susceptible to the same explanation. The treatment for these cases is to divide the external urinary meatus by the side of the trænum down to the level of the coronal sulcus, gently dilate the urethra and then insert a plug of vaseline gauze into the navicular fossa. The meatotomy must be carefully supervised during the succeeding month. In the female child a mild vulvitis is often present. This is often not $c_{5}$ associated with vaginitis. On inspection one sees redness of the vestibule. This is ${ }^{0}$ the locality which lies between the labia minora and the hymen. Such a child $\vec{\oplus}$ nearly always has urethritis. This is generally quite obvious from the appearance of the external urinary meatus. Often enough, there is little or no discharge in such case. The organisms are usually coliform bacilli and staphylococci, and occasionally there is diphtheritic infection. These cases respond to lavage with a mild antiseptic lotion such as lysol $\mathrm{I} / 600$. If the urethra is still reddened after several weeks of treatment, an anæsthetic must be given and dilatation carried out. Occasionally one finds that little girls have gonococcal cervicitis and urethritis. A girl approaching puberty may have leucorrhœa, with frequency of micturition, the result of the discharge getting into the urethra. It is striking how a regular vaginal douche with normal saline helps this condition.

Cervicitis, both simple and gonococcal, are occasionally seen even in young children. They respond best to packing the vagina from time to time with gauze soaked in glycerine. It is not difficult to inspect and to treat the cervix in young $₹$

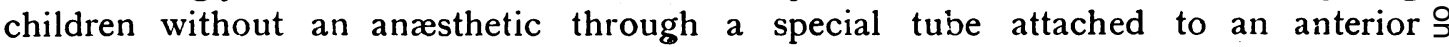
urethroscope.

As to chronic B. coli infection in children who suffer from frequency, I have catheterized the ureters of many of the chronic cases and have found the infection not in the kidneys but in' the bladder as with adults. In children threadworms should be $\stackrel{N}{N}$ sought with the greatest care in puzzling cases of frequency. Dilatation of different parts $\omega$ of the urinary tract leading first to infection and then to frequency accounts for a certain number of cases. Cystoscopy and pyelography are generally necessary to establish this cause. Both sexes are involved. Chronic nephritis will explain some cases of frequency in children. Children with anterior poliomyelitis or spina bifida occulta may suffer from frequency because the nerve supply of the bladder has been interfered with. In the latter condition this is generally an overflow from retention. 
In conclusion one would say that the causes of frequency are not so mysterious if proper investigation is made, and that ascending infection is the commonest cause and my experience is that most cases are amenable to treatment.

I have said practically nothing about drugs for urinary tract infections; I can sum them up briefly and quickly. There is no such thing as an efficient urinary antiseptic. Most of the preparations under this heading can be shown to influence odd cases favourably, but in many others again they cause increased irritation in the urinary tract, and sometimes hæmaturia. There can be no prospect of a beneficial influence on an infection which is often wrongly thought to be seated in the kidney, but which is actually buried under the mucous membrane in some part of the lower urinary tract. Vaccines with occasional exceptions are quite useless. Broadly speaking the proper principle of treatment to follow is regular daily copious draughts of diuretic fluids combined with alkalies.

\section{CASES DEMONSTRATED AT THE M.R.C.P. CLASS.}

\section{A Case of Chronic Rheumatic Heart Disease.}

Histcry. - The patient, Mr. W. C., aged 34, was referred to me in January, I933, with severe heart failure. He had rheumatic fever at the age of 13 , when a heart lesion was recognized, and there have been several recurrences of acute rheumatism. He has led a normal life until a year ago, when increasing dyspnœa prevented him continuing his work. He has been in hospital with congestive heart failure recently.

Examination.- $\mathrm{He}$ is cyanosed and breathless at rest. The veins in the neck are moderately engorged, and pulsate excessively. The pulsation in the neck is mainly venous, but there is also some arterial throbbing. The pulse is completely irregular and characteristic of auricular fibrillation, and is somewhat collapsing in character. Bloodpressure, $130 \mathrm{~mm}$. systolic. The apex beat is in the sixth interspace, in the anterior axilla, and heaving in character. There is a purring systolic thrill at the aortic area, transmitted to the vessels of the neck. On auscultation, there is a loud rough aortic systolic murmur, the aortic second sound is inaudible, and a blowing diastolic murmur is heard along the left of the sternum. These loud aortic murmurs are audible over the whole præcordia. Careful auscultation reveals a distinct diastolic rumble, due to mitral stenosis, at a limited area at the apex, and at the lower end of the sternum a blowing systolic murmur of tricuspid regurgitation.

The liver is considerably enlarged, and pulsates quite noticeably, and there is a moderate degree of ascites. The patient is practically free from œedema, and the lungs show only a few râles at the bases. X-ray examination shows a greatly enlarged heart, which has the characteristic triangular shape of chronic heart failure with tricuspid regurgitation. The right auricle is much enlarged, the superior vena cava is dilated, and the pulmonary artery is prominent. In the oblique position, the left auricle was seen to be enlarged.

The diagnosis is rheumatic heart disease, aortic stenosis and incompetence, mitral stenosis, tricuspid regurgitation, congestive heart failure. It is not possible to say whether the tricuspid regurgitation is due to a rheumatic valvulitis or to chronic dilatation of the right heart. Rheumatic tricuspid disease is not very rare, and is invariably associated with mitral disease ; some degree of stenosis is common in tricuspid 